\title{
L'instabilité chromosomique des premières mitoses blastomériques
}

L'examen du caryotype de villosités choriales prélevées à des fins de diagnostic prénatal montre une plus grande fréquence de trisomies mosaïques que chez les nouveaunés. Il est probable que des phénomènes de non-disjonction ou de retard à l'anaphase, fréquents durant les premières divisions blastomériques, tendent souvent à rétablir la diploïdie de cellules zygotiques, au départ totalement trisomiques. Selon le chromosome perdu au cours de ce processus, le fotus possédera des chromosomes provenant des deux parents ou bien d'un seul parent (disomie uniparentale, associée parfois à des anomalies de développement). Même dans le cas où le fotus est diploïde avec des chromosomes biparentaux, une trisomie placentaire isolée peut entraîner des troubles de la croissance fœetale.

\section{André Boué}

\section{ADRESSE}

A. Boué : professeur directeur de l'Inserm U. 73. Inserm U. 73, génétique et pathologie foetale, château de Longchamp, bois de Boulogne, 75016 Paris, France.

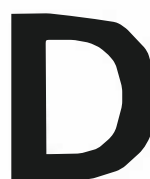

u fait de leur fréquence, des mécanismes en cause et des conséquences, sur le devenir des différentes cellules du blastocyste et, plus généralement, sur le développement, les mosaïques chromosomiques constituent un domaine de recherche d'un très grand intérêt.

Par définition, une mosaïque est la présence chez un même individu de deux ou plusieurs populations cellulaires ayant des compléments chromosomiques différents. Le plus souvent, coexistent une population cellulaire de caryotype normal et une ou plusieurs populations cellulaires avec une ano- malie du nombre de chromosomes, en général une trisomie.

\section{Les mécanismes de formation des mosaïques}

Les mosaïques résultent d'accidents mitotiques survenant au cours des premières divisions après la fécondation.

1. La cellule originelle a un caryotype normal. Selon le stade où survient l'accident mitotique, on trouve, en proportion variable, des cellules normales, des cellules trisomiques et les cellules monosomiques 


\section{RÉFÉRENCES}

1. Crane JP, Cheun SW. An embryogenic model to explain cytogenetic inconsistencies observed in chorionic villus versus fetal tissue. Prenat Diagn 1988 ; 8 : 119-30.

2. Hassold T. Mosaic trisomies in human spontaneous abortions. Hum Genet 1982 ; $61: 31-5$

3. Plachot M, de Grouchy J, Cohen J, Salat-Baroux J. Anomalies chromosomiques de l'œuf humain fécondé. Reprod Nut Dev $1990 ; 1$ (suppl) : 83s-8.

4. Stengel-Rutkowski S, Nimmermann C. Pränatale Diagnose an Chorionzotten in der Bundesrepublik Deutschland. Rapport interne, Munich, 1991.

5. Kalousek DK, Dill FJ. Chromosomal mosaicism confined to the placenta in human conceptions. Science 1983; 221 : 665-7.

6. Kalousek DK, Dill FJ, Pantzar TJ, McGillivray BC, Yong SL, Wilson RD. Confined chorionic mosaicism in prenatal diagnosis. Hum Genet 1987; 77 : 163-7.

7. Kalousek DK, Barrett IJ, McGillivray $\mathrm{BC}$. Placenta mosaicism and intrauterine survival of trisomy 13 and $18 . \mathrm{Am}$ J Hum Genet 1989 ; 44 : 338-43.

8. Kalousek DK, Barrett IJ, Gartner AB. Spontancous abortion and confined mosaicism. Hum Genet 1982 ; 72 : 842-6.

9. Kalousek DK, Howard-Peebles PN, Magenis RE, et al. Confirmation of CVS mosaicism in term placentae and high frequency of intrauterine growth retardation : association with confined placenta mosaicism. Prenat Diagn 1981; 11: 743-50.

10. Appelman Z, Rosensaft J, Chemke J, Caspi B, Astikenazi H, Mogilner MB. Trisomy 9 confined to the placenta : prenatal diagnosis and neonatal follow up. $A m \mathrm{~J} \mathrm{Med}$ Genet 1991 ; 40 : 464-6.

11. Breed ASPM, Mantingh A, Vosters R, Beekhuis JR, Van Lith JMM, Anders GPJA. Follow-up and pregnancy outcome after a diagnosis of mosaicism in CVS. Prenat Diagn 1991; 11 : 577-80.

12. Casey J, Ketterer DM, Heisler KL, Daugherty EA, Prince PM, Giles HR. Prenatal diagnosis of trisomy 2 mosaicism confirmed in foreskin fibroblasts. $\mathrm{Am} \mathrm{J} \mathrm{Hum}$ complémentaires. Cette éventualité est rare car les lignées cellulaires monosomiques, le plus souvent non viables, disparaissent. Persiste donc, le plus souvent, un mélange de cellules normales et de cellules trisomiques.

2. La cellule originelle a un caryotype anormal, en général une trisomie. Au cours des premières divisions, il y a perte d'un des trois chromosomes par retard à l'anaphase (anaphase lagging) ou par nondisjonction, conduisant à une lignée cellulaire diploïde. Selon le chromosome perdu, la lignée cellulaire diploïde retient alors dans cette paire chromosomique, soit un chromosome de chacun des deux géniteurs, soit deux chromosomes d'un même géniteur, c'est-à-dire une disomie uniparentale, et, selon l'accident méiotique initial responsable, soit les deux chromosomes sont identiques (isodisomie), soit ils sont différents (hétérodisomie).

C'est ce deuxième mécanisme, dans lequel la cellule originelle est anormale et où il y a perte du chromosome supplémentaire, qui serait le plus fréquent (80\% des mosaïques).

\section{Les premiers stades du développement (figure 1)}

Il est important de reprendre la séquence des premiers stades du développement embryonnaire pour comprendre l'origine des différentes populations cellulaires embryonnaires et extra-embryonnaires. Cela permet d'expliquer les différentes localisations des mosaïques et la probabilité de leur détection ou de leur méconnaissance selon les prélèvements étudiés. Les cellules des deux couches du trophoblaste s'individualisent très tôt et sont le siège d'une intense prolifération cellulaire, ce qui permet l'analyse directe des mitoses dans la couche du cytotrophoblaste ; celui-ci est à l'origine du syncytiotrophoblaste, où ne se produisent pas de mitoses.

Parallèlement s'individualisent les cellules qui donnent le bouton embryonnaire (inner cell mass) conduisant à plusieurs populations cellulaires distinctes : (a) les cellules du mésoderme extra-embryonnaire qui vont donner le chorion et coloniser la lumière interne des villosités choriales (ces cel- lules prolifèrent in vitro lorsqu'on met en culture les villosités choriales alors que les cellules du syncytiotrophoblaste et du cytotrophoblaste ne se divisent pas in vitro); (b) les cellules du lécithocèle (vésicule vitelline); (c) les cellules de l'amnios; (d) les cellules de l'embryon lui-même [1]. Les cellules qui sont analysées lors des diagnostics chromosomiques sur liquide d'amniocentèse sont en général un mélange de cellules de l'amnios et de cellules de l'embryon provenant de la peau, de l'arbre urinaire, et des voies respiratoires et digestives supérieures.

On voit donc qu'en fonction du prélèvement et, pour les villosités, de la technique directe ou indirecte de l'analyse chromosomique, on étudie les cellules filles d'un même zygote originel mais qui se sont séparées très tôt au cours de l'embryogenèse et donc, dans le cas de mosaïque, qui peuvent ne pas être représentatives du statut chromosomique de l'embryon lui-même.

\section{Quelle est la fréquence des mosaïques?}

A la naissance, on connaît la fréquence des mosaïques dans certains syndromes pathologiques : parmi les enfants trisomiques 21 , on observe des mosaïques dans environ $2 \%$ des cas ; dans les syndromes de Turner*, il y a deux tiers de mosaïques. Quelques études cytogénétiques systématiques ont montré que $0,1 \%$ des nouveau-nés sont en fait des mosaïques. Mais ce sont des études anciennes portant sur l'analyse d'un nombre limité de cellules.

Dans les avortements spontanés, des mosaïques sont décelées dans $5 \%$ des cas. Toutefois, l'étude cytogénétique a porté sur les cellules extraembryonnaires, soit en raison de l'absence de formation embryonnaire, soit par manque de prolifération cellulaire des embryons macérés. L'interprétation de ces résultats est donc difficile [2].

Les analyses cytogénétiques des embryons humains obtenus par fécondation in vitro ont montré la

* Sujets de phénotype féminin, de petite taille et stériles, de caryotype $45 X$. 


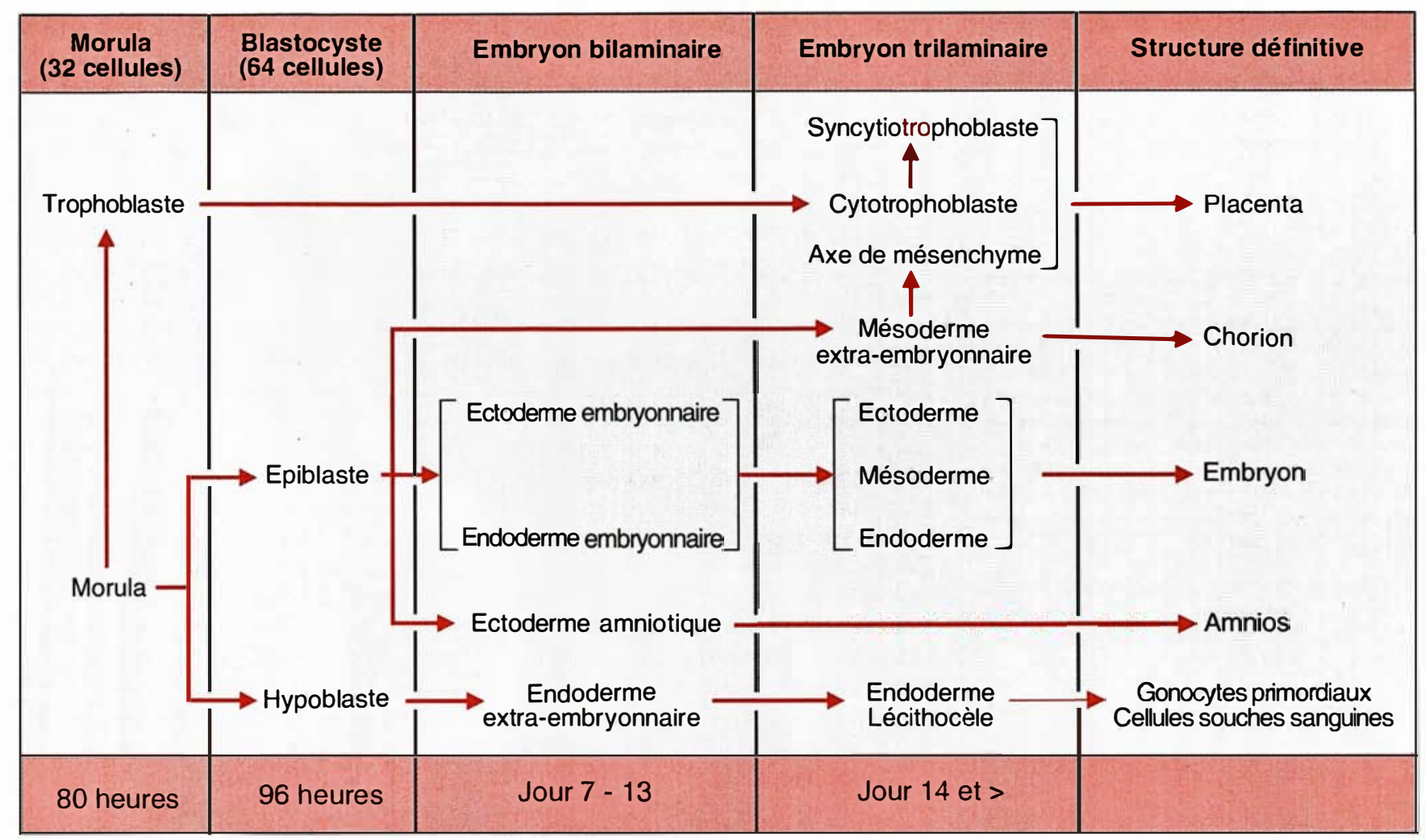

Figure 1. Devenir des cellules du zygote au cours des deux premières semaines du développement. (D'après [1].)

grande fréquence des anomalies chromosomiques [3]: $28 \%$ dans les embryons qui seraient replacés in utero et $42 \%$ des embryons d'aspect dégénératif. Le taux des mosaïques atteint en moyenne $10 \%$ (6 à $11 \%)$.

On voit donc que la fréquence des mosaïques chromosomiques est importante dès les premiers stades du développement du zygote et que la diminution apparente de cette fréquence doit être le résultat, soit du caractère létal de certaines mosaïques si elles concernent l'embryon luimême, soit de la disparition de la lignée cellulaire aneuploïde.

\section{Problèmes diagnostiques causés par l'existence de mosaïques}

Dans les diagnostics prénatals réalisés sur cellules du liquide amniotique, la découverte de mosaïques rendait parfois délicate la conduite à tenir. La technique directe d'analyse chromosomique sur les cellules du cyto$\mathrm{m} / \mathrm{s} n^{\circ} 9 \mathrm{vol} .8$, novembre 92 trophoblaste a révélé une fréquence plus élevée de mosaïques diverses qui posent donc des problèmes d'interprétation encore plus complexes.

Une vaste étude, portant sur les diagnostics prénatals par prélèvement de villosités choriales, a été entreprise en Allemagne entre le $1^{\text {er }}$ mai 1985 et le 21 mars 1990, coordonnée par Sabine Stengel-Rutkowski [4] ; 16 centres ont participé à cette étude qui porte sur 9235 diagnostics. Une analyse très minutieuse des conditions de prélèvement, de la poursuite de la grossesse et de l'étude de l'enfant à la naissance est associée à une étude détaillée des analyses cytogénétiques et des examens anatomopathologiques, lors des interruptions spontanées ou médicales de grossesse.

Le document présente en détail chaque observation posant un problème Il n'existe pas d'autres études équivalentes dans les publications réunies à ce jour.

L'analyse des résultats cytogénétiques est la plus instructive car elle est remarquablement documentée.

L'étude cytogénétique a été réalisée soit directement sur les cellules du trophoblaste, le jour du prélèvement ou après une nuit d'incubation, soit après culture cellulaire de la villosité, soit en combinant ces techniques. En pratique une seule technique est utilisée dans $53 \%$ des cas (immédiate $6 \%$, après incubation $42 \%$, après culture $5 \%$ ), deux techniques dans $43 \%$ (direct + incubation $5 \%$; incubation + culture $38 \%$ ) et les trois techniques dans $3 \%$. On voit donc que dans environ $40 \%$ des examens, une culture cellulaire est pratiquée, ce qui alourdit beaucoup les analyses chromosomiques.

Dès qu'une discordance, une difficulté d'interprétation (mosaïque) ou une anomalie chromosomique exceptionnelle chez le nouveau-né est décelée dans les villosités, une étude cytogénétique des cellules prélevées par amniocentèse est pratiquée. 


\section{RÉFÉRENCES}

13. Delozier-Blanchet CD, Engel E, Extermann P, Pastori B. Trisomy 7 in chorionic villi : follow-up studies of pregnancy, normal child, and placenta clonal anomalies. Prenat Diagn 1988; 8 : 281-6.

14. Fryburg JS, Dimaio MS, Mahoney MJ. Postnatal placental confirmation of trisomy 2 and trisomy 16 detected at chorionic villus sampling. A possible association with intrauterine growth retardation and elevated maternal serum alpha-fetoprotein. Prenat Diagn 1992 ; 12 : 157-62.

15. Guerneri S, Fortuna R, Romitti L, Bettio D, Simoni G. Seven cases of trisomy 3 mosaicism in chorionic villi. Prenat Diagn $1989 ; 9: 691-5$

16. Hashish AF, Monk NA, Lovell Smith MP, Bardwell LM, Fiddes TM, Gardner RJ. Trisomy 16 detected at chorionic villus sampling. Prenat Diagn 1989; 9 : 427-32.

17. Kennernecht I, Terinde R. Intrauterine growth retardation associated with chromosomal aneuploidy confined to the placenta. Three obscrvations : triple trisomy 6, 21, 22 ; trisomy 16 ; and trisomy 18. Prenat Diagn 1990 ; 10 : 539-44.

18. Leschot $\mathrm{NJ}$, Wolf $\mathrm{H}$. Is placental mosaicism associated with poor perinatal outcome? Prenat Diagn 1991; 11 : 403-4.

19. Reddy KS, Blakemore KJ, Stetten G, Gorson V. The significance of trisomy 7 mosaicism in chorionic villus cultures. Prenat Diagn $1990 ; 10: 417-23$.

20. Stioui S, De Silvestris M, Molinari A, Stripparo L, Ghisoni L, Simoni G. Trisomic 22 placenta in a case of severe intrauterine growth retardation. Prenat Diagn $1989 ; 9: 673-6$

21. Watson JD, Ward BE, Peakman D, Henry G. Trisomy 16 and 12 confined chorionic mosaicism in liveborn infants with multiple anomalies. Am J Hum Genet 1988 ; 43 : A251.

22. Williams J, Wang B, Rubin C, Clark R, Mohandas T. Apparent nonmosaic trisomy 16 and chorionic villi : diagnostic dilemma or clinically significant finding? Prenat Diagn $1992 ; 12$ : 157-60.

23. Johnson A, Wapner TJ, Davis GH, Jackson LG. Mosaicism in chorionic villus sampling : an association with poor perinatal outcome. Obstet Gynecol 1990 ; 75 : 573-80.

24. Hall JC. Genomic imprinting and its clinical implications. $N$ Engl J Med 1992 ;
Quels ont été les problèmes rencontrés?

- Anomalies homogènes détectées par analyse directe $d u$ cytotrophoblaste

1. Parmi les anomalies compatibles avec le développement à terme, 123 trisomies 21 ont été décelées. Aucune erreur n'a été relevée lors des contrôles effectués après interruption dans environ la moitié des observations.

Sur 49 trisomies 18, homogènes dans les cellules des villosités, l'analyse des tissus fœtaux, faite dans les deux tiers des cas, a montré un seul faux positif. De même, sur 28 monosomies $\mathrm{X}$ (syndromes de Turner), un faux positif fut observé.

Enfin, deux anomalies de structure, une translocation $21-21$ et une délétion du chromosome $\mathrm{X}$ étaient seulement présentes dans les cellules du trophoblaste, l'embryon ayant un caryotype normal.

2. Un certain nombre d'anomalies exceptionnelles chez les nouveau-nés ont conduit à réaliser une amniocentèse qui a montré un caryotype normal confirmé à la naissance (deux trisomies 7, deux trisomies 16, deux tétraploïdies). Dans un cas de trisomie 6, une interruption a été pratiquée, mais le fœtus avait un caryotype normal.

Quelques faux négatifs ont été observés au cours de ces analyses : une trisomie 21 à la naissance alors qu'un caryotype masculin normal avait été trouvé sur un grand nombre de cellules étudiées par technique directe sur villosités.

Quatre discordances sont par ailleurs très instructives : à l'examen direct ou après incubation, un caryotype normal mâle avait été trouvé, mais, après culture, une anomalie chromosomique a été décelée (deux trisomies 21 , une trisomie 18 , une monosomie X). La culture des villosités a donc permis d'éviter quatre faux négatifs. Dans ces cas, la population cellulaire du cytotrophoblaste était homogène, mais son caryotype était différent de celui de l'embryon.

Ces résultats ont conduit de nombreux centres à associer régulièrement examen, direct ou après incubation, et culture de villosités.

- Mosaïques décelées par un examen direct (au total 142 observations, soit environ $1,5 \%)$
En pratique, en dehors des trisomies 18 et 21 , toutes les mosaïques étaient seulement présentes dans les cellules des villosités et le caryotype des cellules prélevées par amniocentèse, ou dans quelques cas après interruption, était toujours normal. En revanche, sur six mosaïques avec trisomie 18, la moitié comportaient une trisomie 18 homogène dans les cellules amniotiques et dans les cellules fœtales, et les trois autres cas avaient un caryotype normal confirmé à la naissance ; parmi les quatre mosaïques avec trisomie 21, une trisomie 21 homogène fœtale était décelée et trois fœtus étaient normaux. On doit donc être particulièrement vigilant dans les cas des trisomies 18 et 21 .

L'analyse détaillée de quelques observations est présentée dans les figures 2 à 5 .

Cette remarquable étude apporte d'importantes précisions sur les difficultés de l'analyse chromosomique sur villosités. Si l'examen direct avait été la seule technique d'analyse chromosomique, il y aurait eu cinq faux négatifs, onze faux positifs comportant des anomalies homogènes (six pouvant être considérées comme douteuses car exceptionnelles à la naissance) et 142 mosaïques demandant une amniocentèse.

Si l'on veut se mettre à l'abri des erreurs, il faut associer l'examen direct (immédiat ou après incubation) et l'examen sur les cellules de villosités en culture. Il faut également, au moindre doute, contrôler le diagnostic par un examen des cellules prélevées par amniocentèse. La fiabilité est à ce prix, et donner un diagnostic sur le seul examen direct implique d'accepter un certain nombre d'erreurs.

\section{Les mosaïques au niveau du placenta peuvent-elles expliquer certaines anomalies}

L'utilisation de la technique directe d'analyse chromosomique sur les cellules du cytotrophoblaste a introduit la notion de mosaïque confinée au placenta (confined placental mosaicism) $[5,6]$.

Des études cytogénétiques réalisées par Kalousek à Vancouver [7], sur le

$$
m / s n^{\circ} 9 \text { vol. } 8 \text {, novembre } 92
$$




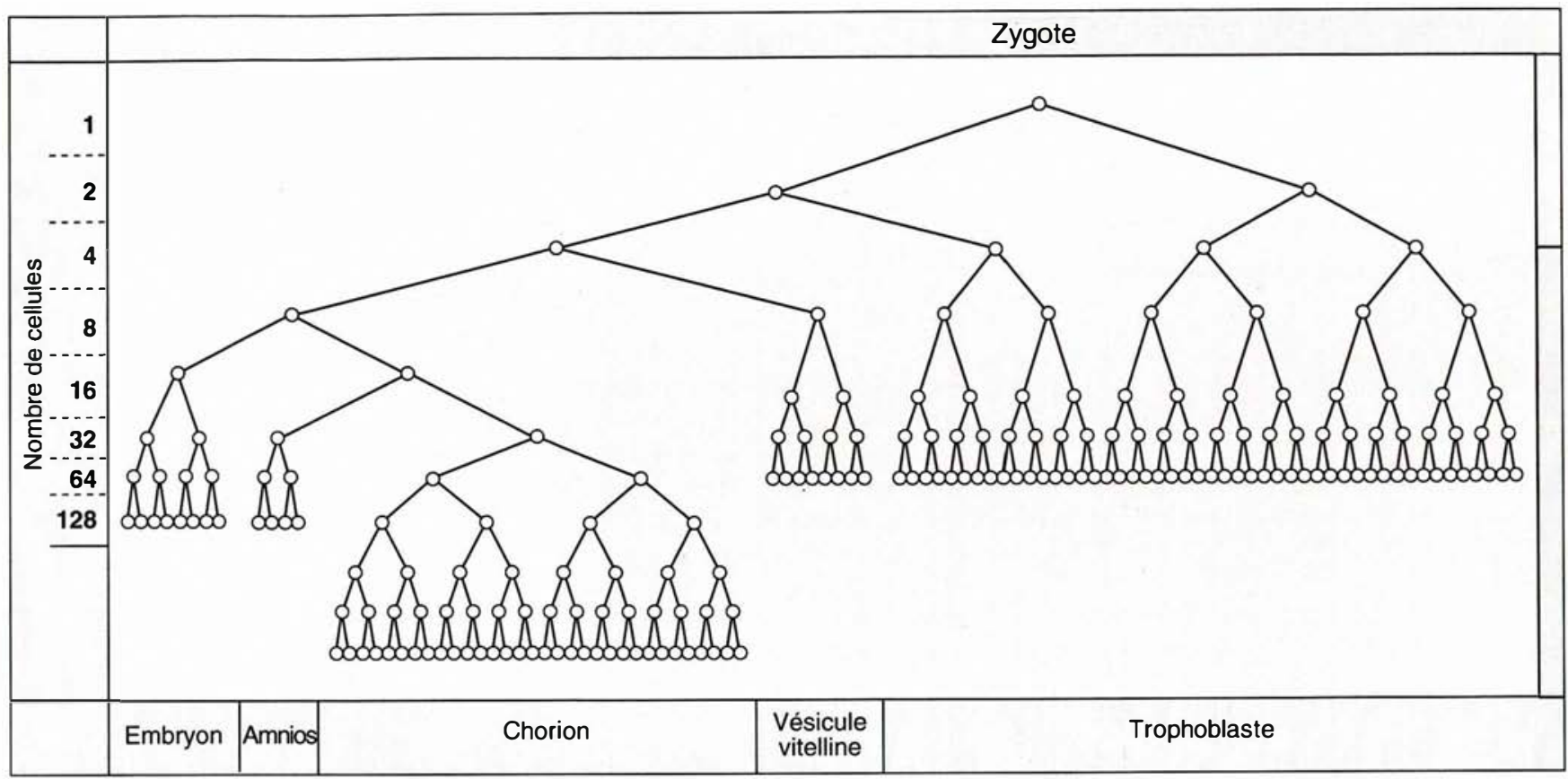

Figure 2. Premiers cycles de divisions cellulaires au cours de l'embryogenèse normale. (D'après [1] modifié par S. Stengel-Rutkowski.)

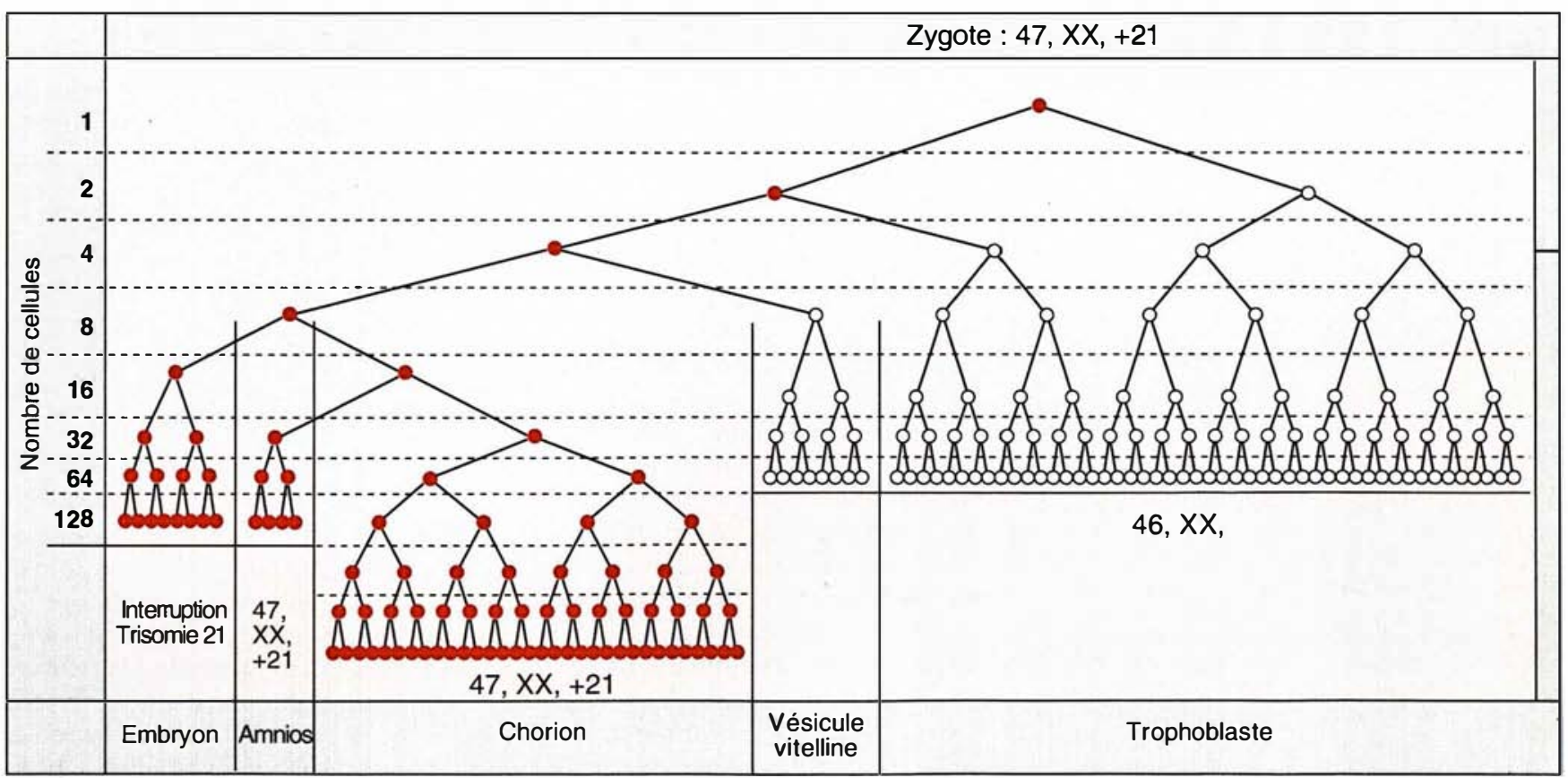

Figure 3. Observation d'un résultat faussement négatif. Le caryotype réalisé directement sur les cellules cytotrophoblastiques est normal, alors qu'une analyse effectuée après culture du trophoblaste montre une trisomie 21 homogène, confirmée par l'analyse des cellules du liquide amniotique et des tissus fœtaux après interruption de la grossesse. (D'après S. Stengel-Rutkowski.) 


\section{RÉFÉRENCES}

25. Cassidy SB, Lai LW, Erickson RP. Trisomy 15 with loss of the paternal 15 as a cause of Prader Willi syndrome due to maternal disomy. Am J Hum Genet 1992 (sous presse).

26. Purvis-Smith SG, Saville T, Manass S, et al. Uniparental disomy 15 resulting from correction of an initial trisomy 15. $A m \mathrm{~J}$ Hum Genet 1992 ; 50 : 1348-50.

27. Morichon-Delvallez $\mathrm{N}$, Mussat $\mathrm{P}$, Dumez Y, Vekemans M. Trisomy 15 in chorionic villi and Prader Willi syndrome at birth. Prenat Diagn 1992 (sous presse).

28. Kalousek DK, Langlois S, Barrett I, et al. Uniparental disomy from chromosome 16 in humans. VIth International Conference on early prenatal diagnosis of genetic diseases, Milan, 1992.

29. Babinet C. L'empreinte génomique parentale. médecine/sciences $1992 ; 8: 65-70$.

30. Szulman AE, Philippe E, Boué JC, Boué A. Human triploidy : association with partial hydatiform moles and non molar conceptuses. Hum Pathol 1981 ; 12 : 1016-21.

31. Jacobs PA, Szulman AE, Funkhouser J, Matsuura JS, Wilson CC. Human triploidy : relationship between parental origin of the additional haploid complement and development of partial hydatiform mole. $\mathrm{Am}$ Hum Genet 1982 ; 46 : 223-31.

32. Adinolfi M. On a non-invasive approach to prenatal diagnosis based on the detection of a fetal nucleated cells in maternal blood samples. Prenat Diagn 1991; 11 : 794-804.

33. Szollosi D. The spindle structure in mammalian eggs : the effect of aging. In : Boué A, Thibault C, eds. Les Accidents chromosomiques de la reproduction. Paris : Éditions Inserm, 1973 : 241-50.

34. Oberlé I, Mandel JL. Retard mental avec $\mathrm{X}$ fragile : une empreinte génomique très localisée, étroitement liée à l'expression clinique. médecine/sciences $1991 ; 7$ : 378-9.

35. Junien C, Lavedan C. Dystrophie myotonique de Steinert: encore une mutation instable. médecine/sciences $1992 ; 8: 249-51$.

36. Devys D, Biancalana V, Rousseau F, Boué J, Mandel JL, Oberlé I. Analysis of full fragile $X$ mutations in fetal tissues and monozygotic twins indicate that abnormal methylation and somatic heterogeneity are established early in development. $\mathrm{Am} \mathrm{J} \mathrm{Med}$ placenta de trisomies 13 et de trisomies 18 ayant évolué jusqu'à terme, ont montré que, dans toutes les observations, il y avait une constitution en mosaïque du placenta, alors que le nouveau-né avait une trisomie homogène. En moyenne, deux tiers des cellules cytotrophoblastiques (permettant une analyse cytogénétique directe) étaient diploïdes. On peut penser que l'activité hormonale de ces cellules trophoblastiques diploïdes a permis l'évolution de la grossesse à terme. Une hypothèse identique peut être faite pour les monosomies X ; dans les avortements spontanés, les monosomies $\mathrm{X}$ sont homogènes, mais on sait que dans le syndrome de Turner deux tiers des sujets atteints sont mosaïques, $45 \mathrm{X}$ associé le plus souvent à un caryotype diploïde. Dans ces cas, une constitution en mosaïque du placenta peut aussi expliquer le développement jusqu'à terme.

De récents travaux de la même équipe [8] portant sur des avortements spontanés ont montré une fréquence de mosaïques confinées au placenta plus élevée que celles rapportées dans les études antérieures fondées uniquement sur des analyses chromosomiques réalisées sur des cellules en culture.

D'autres travaux de la même équipe [9] ont porté sur le devenir d'embryons ayant un caryotype normal dont le placenta comportait un fort pourcentage de cellules aneuploïdes (en général une population cellulaire trisomique). Ces aneuploïdies placentaires avaient été décelées lors de diagnostics prénatals par analyse directe de villosités choriales et confirmées par l'examen cytogénétique du placenta à la naissance (une analyse des cellules amniotiques ayant démontré le caryotype normal du fœtus). Dans ce groupe, une proportion importante de retard de croissance intra-utérine a été observée, sans syndrome malformatif, mettant en relief une déficience du fonctionnement placentaire lorsqu'il existe une population importante de cellules aneuploïdes.

Une série de publications récentes [10-22] rapportent des observations de cellules trisomiques au niveau du placenta, mais avec caryotype normal d'après l'analyse des cellules du liquide amniotique. Dans certaines observations, la grossesse étant pour- suivie, un important retard de croissance était observé ; mais l'évolution post-natale a été favorable dans l'ensemble. Johnson [23], sur une grande série de 4319 diagnostics effectués sur villosités choriales, rapporte 55 mosaïques au niveau du placenta et une augmentation importante de la mortalité pré- et périnatale dans ce groupe $(16,7 \%$ contre $2,3 \%)$.

\section{Mosaïques et disomie uniparentale}

Les sujets porteurs d'une disomie uniparentale ont un complément chromosomique normal, mais, pour une paire d'autosomes, les deux chromosomes proviennent d'un même géniteur. Ces deux chromosomes peuvent être soit différents (nondisjonction à la première division méiotique), soit identiques (isodisomie par duplication à la deuxième division méiotique) $\left(\mathrm{m} / \mathrm{s} n^{\circ} 1\right.$, vol. 6 , p. 57).

Dans un premier temps, on a supposé que ces disomies uniparentales résultaient de la fusion d'un gamète disomique avec un gamète nullisomique pour la même paire chromosomique. La fréquence des anomalies numériques de non-disjonction dans les gamètes, qui a surtout pour conséquence des zygotes monosomiques et trisomiques éliminés précocement, pouvait expliquer la survenue fortuite de ces zygotes avec disomie uniparentale. Mais la fréquence des mosaïques observées dans les diagnostics sur villosités choriales et sur les embryons in vitro conduit à penser que l'éventualité la plus probable est celle d'un zygote trisomique où l'embryon est issu d'un clone cellulaire ayant perdu l'un des trois autosomes.

On connaît des exemples bien documentés de disomies uniparentales [24].

Chez deux enfants atteints de mucoviscidose, l'anályse moléculaire a montré, d'après les polymorphismes voisins du gène de la mucoviscidose et d'autres polymorphismes répartis sur tout le chromosome 7, que les deux chromosomes 7 étaient identiques à l'un des chromosomes maternels. Ces deux enfants présentaient également une petite stature en rapport probablement avec l'homozygo- 


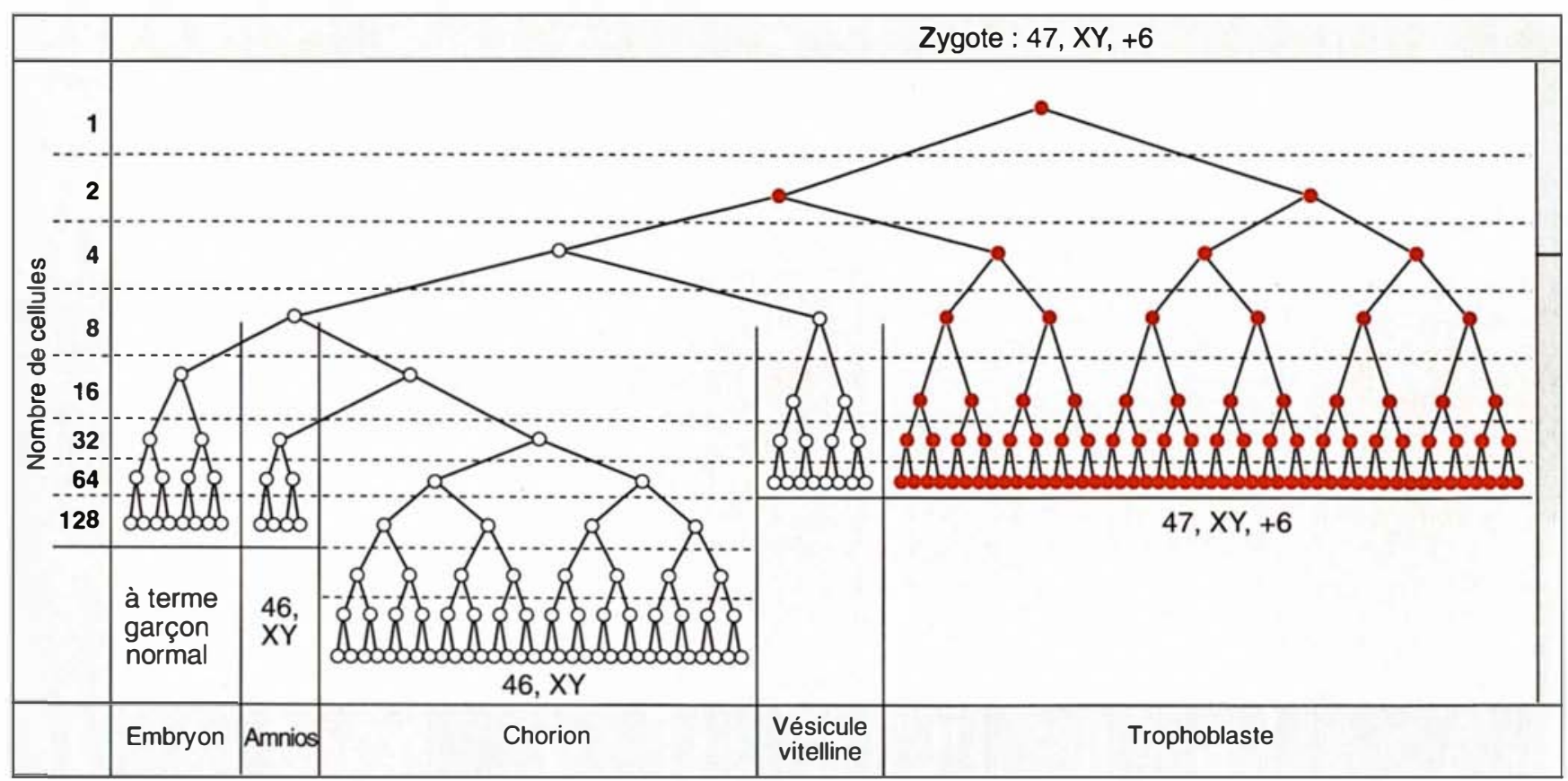

Figure 4. Observation d'un résultat faussement positif. Une trisomie 6 homogène d'après l'analyse directe des cellules du cytotrophoblaste, un caryotype normal des cellules cultivées et du liquide amniotique et un enfant normal. (D'après S. Stengel-Rutkowski.)

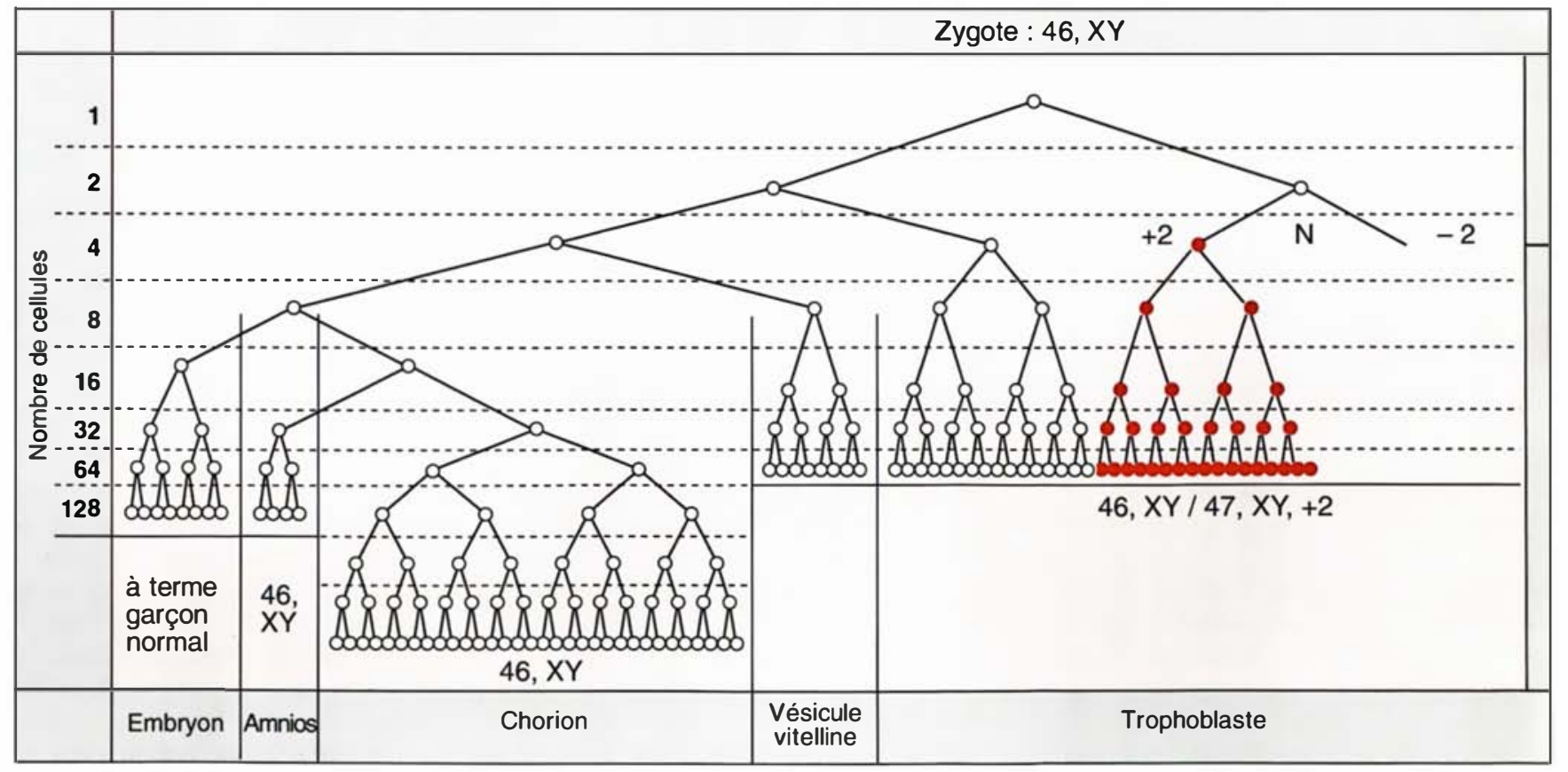

Figure 5. Observation d'une mosaïque au niveau du cytotrophoblaste. Trisomie 2/normal (les cellules avec la monosomie 2 ont disparu). Les cellules en culture du trophoblaste et du liquide amniotique sont normales. ( $D$ 'après S. Stengel-Rutkowski.) 
tie d'autres gènes sur le chromosome 7.

Deux syndromes malformatifs associés à un retard mental, le syndrome Prader Willi et le syndrome d'Angelman, ont des expressions phénotypiques très différentes. Dans ces deux syndromes, on a mis en évidence (dans la majorité des cas) une délétion sur le grand bras du chromosome 15 , près du centromère (15q11q13). L'analyse moléculaire confirme cette large délétion qui, dans les deux syndromes, implique le même segment d'ADN.

Dans le syndrome de Prader Willi, la délétion est située sur le chromosome 15 d'origine paternelle, dans le syndrome d'Angelman sur le 15 d'origine maternelle. Ainsi la complémentarité de cette région chromosomique, paternelle ou maternelle, est indispensable à un développement normal.

Il existe également des observations de ces syndromes où deux chromosomes 15 sans délétion sont présents, mais ces deux chromosomes 15 parfaitement normaux sont hérités d'un seul des deux parents : disomie uniparentale 15 maternelle dans le syndrome de Prader Willi, disomie 15 paternelle dans le syndrome d'Angelman. Donc la présence de deux chromosomes 15 normaux ne suffit pas à assurer un développement normal, il faut que cette portion d'ADN ait reçu une "empreinte " de chacun des parents pour que le développement soit normal $\left(\mathrm{m} / \mathrm{s} n^{\circ}\right.$ 9, vol. 7, p. 974 et $n^{\circ} 7$, vol. 8, p. 741).

Récemment trois équipes différentes, Cassidy, Purvis-Smith et Morichon, ont rapporté [25-27] des observations identiques particulièrement instructives. Un diagnostic cytogénétique prénatal sur villosités choriales a montré une trisomie 15 homogène, alors que l'analyse des cellules amniotiques concluait à la normalité du caryotype. La grossesse a été poursuivie et, à la naissance, l'enfant présentait un syndrome de Prader Willi, une disomie uniparentale avec deux chromosomes 15 maternels étant ultérieurement prouvée.

Kalousek rapporte huit diagnostics prénatals sur villosités choriales avec, à l'analyse directe, une trisomie 16 (homogène ou en mosaïque) et un embryon diploïde [28].
Une analyse moléculaire à l'aide de marqueurs polymorphes a permis d'identifier les deux chromosomes 16 présents dans les cellules fœtales. Dans quatre observations, il y avait un chromosome 16 paternel et un maternel ; dans un cas, un retard de croissance était observé (poids de naissance $1900 \mathrm{~g}$ ). Dans quatre observations, les deux chromosomes 16 étaient d'origine maternelle ; une mort in utero à 20 semaines, une interruption de grossesse à 25 semaines avec retard de croissance et hypertension maternelle et deux retards de croissance étaient observés dans ces cas.

Sur cette première série bien documentée, on peut penser que la présence du chromosome 16 paternel est nécessaire à un bon développement. On doit rappeler qu'un des premiers exemples d'empreinte génomique [29] a été une anomalie du placenta en relation avec la triploïdie : lorsqu'il y a deux jeux chromosomiques paternels (diandrie dispermie), on observe une prolifération du placenta donnant une môle embryonnée ; lorsqu'il y a deux jeux chromosomiques maternels, l'examen de l'œuf montre une hypoplasie placentaire $[30,31]$.

\section{Conclusion}

Dans la pratique du diagnostic prénatal

L'analyse des cellules du liquide amniotique représente la meilleure technique pour déterminer le caryotype fœtal, car les cellules analysées sont soit des cellules provenant de l'organisme fotal lui-même, soit des cellules des membranes amniotiques proches du fœtus sur le plan de l'embryogenèse.

L'analyse directe du cytotrophoblaste expose à un certain nombre d'erreurs, faux positifs et faux négatifs résultant de mosaïques. Pour pallier ce problème, certains laboratoires font simultanément une analyse des cellules du mésenchyme de la villosité mises en culture. L'association de plusieurs techniques est particulièrement importante lorsque l'âge maternel est élevé, car la fréquence des mosaïques est alors aussi importante que celle des anomalies chromosomiques.

Quelles difficultés d'analyse pourrait représenter l'application de nouvelles techniques de diagnostic prénatal pour la détection des mosaïques ?

En supposant tous les problèmes techniques pratiques résolus (et on en est encore loin !), le diagnostic cytogénétique préimplantatoire pourra être effectué sur une, voire deux cellules. Que représente cette cellule par rapport à la future formation embryonnaire? On risque de se retrouver dans une situation analogue à celle résultant aujourd'hui de l'analyse directe du cytotrophoblaste avec, in fine, une fréquence de mosaïques trois à cinq fois supérieure [3]. Un problème se posera aussi dans le cas des analyses effectuées sur des cellules fœtales du sang maternel. Certaines techniques de sélection des cellules fotales utilisent des anticorps monoclonaux spécifiques des cellules trophoblastiques [32]. L'analyse cytogénétique portera donc sur des cellules identiques à celles qui sont analysées dans les villosités et, dans ce cas, sans le secours d'un contrôle par l'analyse des cellules en culture.

\section{Sur le plan de la pathologie} chromosomique

L'évolution vers la diploïdie de cellules embryonnaires d'un zygote trisomique, par retard à l'anaphase ou par non-disjonction, amène à plusieurs réflexions.

D'abord, l'ampleur du phénomène : si l'on part des résultats des analyses chromosomiques directes sur les villosités choriales [4], on peut estimer que sur 1000 grossesses ayant atteint le stade de 10 semaines, la coexistence d'une trisomie, homogène ou partielle, dans le placenta et d'un embryon diploïde se rencontre dans six cas.

Deux éventualités sont possibles :

- le chromosome supplémentaire perdu provient du gamète disomique; l'embryon, diploïde normal, possède pour la paire d'autosomes en cause un chromosome d'origine paternelle et un d'origine maternelle. On peut donc estimer qu'un nouveau-né sur 250 possédant un caryotype normal est issu d'un zygote qui était trisomique à la conception ; - si le chromosome supplémentaire perdu provient du gamète monosomique, une disomie uniparentale se produit (2 cas sur 1000 grossesses). 
On sait que, pour la trisomie 21, l'accident méiotique de non-disjonction est d'origine maternelle dans $95 \%$ des cas et essentiellement à la première méiose. On devrait donc avoir avant tout des disomies maternelles, le plus souvent des hétérodisomies* et rarement des isodisomies*. Les quelques observations documentées montrent des disomies maternelles [24, 25, 27, 28]. Quelles peuvent être les conséquences d'une trisomie confinée au placenta et d'un embryon avec disomie uniparentale sur le développement? Un placenta trisomique peut avoir des fonctions métaboliques anormales avec un retentissement sur le développement embryonnaire, mais une disomie uniparentale peut aussi avoir des conséquences pathologiques du fait de ses particularités génétiques : (a) arrêt du développement et avortement ; (b) retard de croissance intra-utérin ; ou (c) mortalité périnatale.

D'un point de vue pratique, lors du diagnostic d'une mosaïque avec une trisomie dans le cytotrophoblaste et un caryotype normal de l'embryon, il serait important d'entreprendre une identification des deux chromosomes restants. Les nombreux marqueurs moléculaires (minisatellites en particulier) rendent cet examen possible et permettraient de déceler les disomies uniparentales : le devenir de ces embryons et les données pronostiques dans l'avenir pourraient être ainsi mieux établis.

\section{Sur le plan fondamental}

L'étude sur les avortements spontanés entreprises pendant les années 1960 avaient montré la grande fréquence des anomalies numériques des chromosomes survenant soit au cours des méioses, soit au moment de la fécondation. A cette époque, ces travaux avaient même suscité une certaine incrédulité du fait de l'importance de ce phénomène qui concernait environ une conception sur deux. Les études poursuivies depuis sur les gamètes et les embryons in vitro n'ont fait que confirmer la fréquence des ces erreurs chromosomiques.

\footnotetext{
* Rappel : hétérodisomie uniparentale : les deux chromosomes sont ceux de la paire chromosomique d'un seul parent. Isodisomie uniparentale: les deux chromosomes sont identiques à l'un des chromosomes d'une paire d'un des parents.
}

On se rend compte aujourd'hui que les accidents de non-disjonction chromosomique ne surviennent pas seulement pendant la méiose, mais aussi pendant les premières divisions mitotiques de l'œuf fécondé.

Les travaux de Szollosi [33] avaient montré une absence de centriole dans l'ovocyte des mammifères. En l'absence des centrioles, le fuseau méiotique est moins stable et les chromosomes peuvent s'en éloigner, cela pouvant représenter la base cytologique du phénomène de nondisjonction. Cette absence de centriole est aussi observée lors des premières divisions mitotiques de segmentation et peut donc aussi favoriser les nondisjonctions et les retards à l'anaphase.

Au cours des dernières années, les travaux effectués sur des embryons humains in vitro ont eu surtout des objectifs pratiques tendant à améliorer les techniques de procréation médicalement assistée. Le moment n'est-il pas venu de mener une recherche plus fondamentale sur les premiers stades du développement? Dans ce domaine, on ne connaît pas de modèles animaux présentant la même fréquence d'erreurs chromosomiques et les recherches doivent donc être faites sur des embryons humains.

Peut-on rapprocher de ces mosaïques chromosomiques les mosaïques moléculaires qui ont été récemment mises en évidence dans le syndrome de l'X fragile [34] et dans la myotonie dystrophique de Steinert [35] ? Un fragment d'ADN, segment répétitif CGG dans l'X fragile, CTG dans la myotonie, présente une amplification variable entre une mère porteuse d'une "pré-mutation" (avec une amplification limitée de triplets répétés) et ses enfants atteints. Dans l'X fragile, chez quelques sujets atteints, on observe une constitution mosaïque avec un segment d'ADN ayant conservé la longueur de la prémutation et une série de segments allongés de longueurs différentes. On est conduit à supposer que le zygote conçu n'avait que la prémutation héritée de la mère et que les allongements conduisant à une mutation complète ont été uniquement mitotiques, et cela au cours des premières mitoses blastomériques, comme l'a montré l'étude de jumeaux monozygotes [36].

Ces faits soulignent la nécessité d'effectuer des recherches fondamentales non seulement sur la mécanique chromosomique mais également sur la réplication de l'ARN au cours des premières mitoses blastomériques, sur l'influence des métabolites du cytoplasme et de l'ADN d'origine maternelle qui pourraient être des éléments déterminants de l'empreinte génomique

\section{Summary}

Chromosomal instability of the mitosis of the blastocyst

Chromosomal mosaicism occurs more commonly in prenatal diagnosis in chorionic villus sampling than in liveborn individuals. A German collaborative study on 9000 prenatal diagnosis affords precisc data on the incidence of mosaicisms, their types and the mechanisms involved during the first mitosis of the blastocyst and especially the frequence of anaphase lagging. These mosaicisms lead to confined placental mosaicism with consequences on the development of a diploid embryo, to uniparental disomy, illustrated by recent observations of Prader Willi syndrome. A careful study of similar observations will enlarge this new chapter on the consequences of chromosomal anomalies on fetal development.

\section{TIRÉS A PART}

A. Boué. 